\title{
Worst-Case Vehicle Evaluation Methodology- Examples on Truck Rollover/Jackknifing and Active Yaw Control Systems
}

\author{
WEN-HOU MA* AND HUEI PENG ${ }^{\dagger}$
}

\section{SUMMARY}

\begin{abstract}
A worst-case vehicle evaluation methodology is presented in this paper. This evaluation method identifies worst-case excitation signals so that the vehicle performance under extreme conditions can be assessed. Two case study examples are presented to illustrate the design procedure and potential benefits of this method: the rollover and jackknifing of an articulated truck, and the evaluation of an active yaw control system. In both cases, the worst-case method was able to produce unstable results at very modest steering/braking levels.
\end{abstract}

\section{INTRODUCTION}

Automatic control systems are now an integral part of many modern vehicle systems and have greatly improved their efficiency, safety and convenience. While many tools exist for the design, analysis and simulations of vehicles and vehicle control systems, most vehicle designs have been evaluated/fine-tuned in a brute-force manner. This brute-force evaluation process is time-consuming because one hopes to ensure that these vehicles work satisfactorily under all possible scenarios. The evaluation process usually involves extensive experiments and/or simulations, and may take up to dozens of man-months to accomplish. Further, there is no guarantee that the worst-case condition has been identified. These two weaknesses demonstrate the need for a more systematic vehicle evaluation methodology. Such methodology should identify the weak link of the vehicle designs, and generate worst-case scenarios intelligently.

\footnotetext{
"Mechanical Dynamics Inc.

Assistant Professor, Dept. of Mechanical Engineering and Applied Mechanics, University of Michigan, Ann Arbor, MI 48109-2125, U.S.A. Tel. (734) 936-0352, E-mail: hpeng@umich.edu
} 
A worst-case vehicle evaluation methodology is presented in this paper. The purpose of this evaluation method is to generate excitation inputs (steering, braking, etc.) so that the vehicle performance under worst-case scenarios can be assessed. The worst-case disturbance generation problem is treated as an optimal "game" problem. Classical optimal control and game theories are applied to obtain the worst-case disturbances. Due to the fact that vehicle control systems are becoming more popular on modern vehicles, both 1-player (roughly speaking, no control inputs) and 2-player (with control inputs) cases need to be solved. To demonstrate the design process of the proposed worst-case vehicle evaluation methodology, two vehicle evaluation cases are presented: a truck rollover/jackknife problem and an active yaw control evaluation problem. For both case studies, safety is of utmost concerns, i.e., it makes sense to investigate the worst-case performance of these two problems.

For the first example, the maneuvers to rollover or jackknife an Army Tractor/ trailer (M916A1/M870A2) vehicle is presented. Rollover and jackknifing are responsible for a significant percentage of the accidents and casualties of commercial vehicles. Therefore, many research efforts have been devoted to these two vehicle instability behaviors. In the past, much research has focused on the relationship between these two instability modes and vehicle parameters. For example, it was found [1] that the roll instability is highly correlated to the vehicle lateral acceleration level. When the lateral acceleration is below a certain threshold, the vehicle is usually safe from roll instability, and this bound is not affected by the vehicle longitudinal speed. This acceleration threshold was found to be related to many vehicle parameters, including the ratio between track width and height of the vehicle center of gravity, tire and suspension roll stiffness, location of the vehicle roll center, etc. A detailed discussion on the relationship between tire/suspension characteristic to roll instability can be found in [2].

The yaw instability modes, on the other hand, were found to be affected by both lateral acceleration and vehicle speed [1]. The yaw instability can occur at relatively low lateral acceleration $(0.1-0.2 \mathrm{~g})$ at high vehicle speeds. For highway operations, roll instability can be avoided if we restrict the vehicle lateral acceleration under a certain limit, but the same cannot be said for yaw instabilities. In particular, for jackknife, it was found that when the truck brake system is not designed properly and the trailer axles are locked sooner than the tractor axles under heavy braking, jackknife could occur even when the lateral acceleration is at extremely low levels. While this research pointed out the general relationship between certain vehicle parameters and the potential instability problem, it is not clear how to generate "bad" steering and braking inputs to rollover or jackknife the truck. The latter is an important problem because in order to compare the rollover susceptibility of several vehicles, it is necessary to identify the worst-case excitations for each of these vehicles. Current practice uses the same arbitrarily selected maneuver to excite several vehicles, based on which the rollover characteristics of these vehicles are judged. Apparently, the selected maneuver may favor one vehicle over others and may not be a fair basis for performance assessment. The proposed worst-case vehicle 
evaluation methodology identifies the weak link of each individual vehicle and generates the worst-case maneuvers accordingly. Therefore, the vehicle performance can be more fairly assessed.

While it is relatively easy to figure out how to manipulate a single input, it is not straightforward to coordinate multiple inputs (steering and braking) that complement (rather than fight with) each other. One such coordination scheme has been proposed by UMTRI (The University of Michigan Transportation Research Institute) for the evaluation of passenger cars [3]. They proposed to apply steering inputs that excite the vehicle consistently on one side (half sinusoidal, step, etc.), and apply maximum brake when the vehicle roll rate becomes zero (i.e., the vehicle roll angle peaks on one side), and then switch the brake off when the roll rate becomes zero again (i.e., the vehicle roll angle peaks on the other side). They named this scheme the "drastic maneuver" and successfully applied it to rollover a passenger car.

In this paper, we will present a worst-case scenario generation algorithm which solves the following problem: when the steering and braking inputs of a truck are limited below a certain threshold value, find the input combination which is most detrimental for truck rollover (or jackknife).

The second case study is on the evaluation of an Active Yaw Control (AYC) system. The main objective of AYC systems is to maintain small vehicle side slip angle under all driving conditions. This is achieved through proper control of the vehicle yaw moment. Among various yaw moment control methods, differential braking was found to be very effective and has been the most popular method. AYC function is activated when incipient vehicle spin is detected. AYC devices are also know as Vehicle Dynamics Control (VDC), Vehicle Stability Control (VSC) or Interactive Vehicle Dynamics (IVD), possibly with slightly different design objectives/scopes.

There are two major types of AYC designs: the phase plane approach $[4,5]$ and the simultaneous yaw rate/vehicle side slip control designs [6,7]. The former utilizes the phase plane analysis to characterize the vehicle lateral stability. The control action is activated when the vehicle states enter the unstable regions. This approach requires identifying the boundaries of the stable and unstable region under different steering angles and vehicle speed, which is not a trivial task. The latter approach requires estimations of the desired yaw rate and side slip angle based on driver's inputs and vehicle states. A feedback control algorithm (e.g., PID, LQG) is then designed to reduce the difference between the actual and desired vehicle motion.

AYC is an example of Advanced Vehicle Control and Safety Systems (AVCSS) which apply advanced vehicle control, sensing, and communication techniques to enhance vehicle safety. AVCSS products are expected to enhance vehicle safety, especially under extreme conditions. Therefore, it makes sense to apply the worstcase methodology to evaluate their performance. The evaluation problem is formulated as a "game" problem in this paper. In this two-player game, the control player (the AYC controller) tries to maintain yaw stability, while the disturbance player, which controls the steering and brake pressure, represents the action of a 
human driver. When the disturbance player's action is generated by the worst-case algorithm, the AYC is examined under the worst possible human driver it could expect to see.

This paper is organized as following. In Section 2, the design process of the worstcase vehicle evaluation methodology is summarized. The truck rollover/jackknifing example is presented in Section 3, and the AYC evaluation problem is presented in Section 4. Finally, conclusions are drawn in Section 5.

\section{WORST-CASE EVALUATION METHODOLOGY}

A worst-case vehicle evaluation methodology was recently proposed by Ma and Peng [8] to generate worst-case disturbances for vehicles systems which might include control sub-systems. The design procedure is as follows: first, if the vehicle model is nonlinear, it will be simplified to obtain a linear model. Depending on the number of player(s) and information structure, the game problem is then classified into one of the four cases

- One player without preview information (1P)

- One player with preview information (1PP)

- Two players without preview information (2P)

- Two players with preview information (2PP)

where the one-player cases apply when either no control sub-systems exist, or they exist but their objectives do not conflict with those of the disturbance player. When control sub-systems exist and conflict with the goal of the disturbance player, twoplayer cases should be applied. It is proper to apply the preview cases when significant actuator delays are present.

Solutions of the linear worst-case problems can be obtained analytically, whereas the equations for all the four cases were summarized in [9]. These analytical solutions then serve as initial guess for nonlinear learning (numerical) algorithms. The nonlinear learning algorithm essentially solves a two-point-boundary-value-problem (TPBVP) by using the original nonlinear vehicle model. The TPBVP is solved continuously until an optimal cost function is identified. The worst-case disturbance signal is then obtained from the optimal co-state variables. Numerical methods for solving the four cases are summarized in [10].

\section{TRUCK ROLLOVER/JACKKNIFE PROBLEM}

In this section, the rollover and jackknifing of an articulated truck are used as example one-player worst-case evaluation problems. The worst-case truck rollover/ jackknifing problem is officially stated below: 
Given a nonlinear truck dynamic model $\underline{\dot{x}}=f(\underline{x}, \underline{w}, t)$, where the disturbance input $\underline{w}$ includes front wheel steering angle $\delta$ and brake pressure $p$. Find, within saturation bound $-\delta_{\max } \leq \delta \leq \delta_{\max }$ and $0 \leq p \leq p_{\max }$, the time signal $\underline{w}(t)$ which maximizes $J(\underline{x}, \underline{w}, t)=\int_{0}^{T} \underline{x}^{T}(t) Q \underline{x}(t)-\underline{w}^{T}(t) P \underline{w}(t) d t$ (the cost function). The matrix $Q$ is selected such that the vehicle roll (articulation) motion is maximized in the rollover (jackknifing) problem.

\subsection{Vehicle simulation model}

In this paper, the simulation output of the ArcSim software is assumed to be the true vehicle output. ArcSim is a simulation program developed at the Automotive Research Center (ARC) of the University of Michigan for studying the braking and handling response of the M916A1/M870A2 Army Truck. This truck has a three-axle tractor and a three-axle semi-trailer. In addition to the vehicle dynamics, ArcSim includes an easy to use Simulation and Graphic User Interface (SGUI, see Fig. 1). Furthermore, a plotter and a wire-frame animator are also included. The ArcSim software runs under Windows 95 and Windows NT and is a freeware available at the ARC web site at http://arc.engin.umich.edu/sw_distri/ARCSIM/arcsim.html.

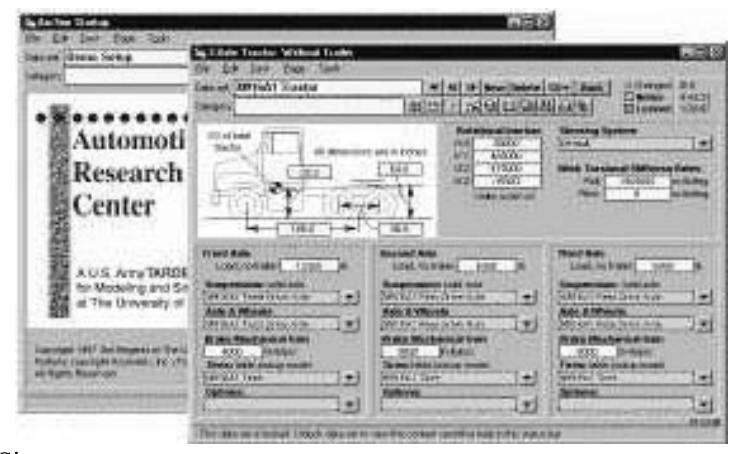

Fig. 1. SGUI of ArcSim.

The ArcSim model includes 33 degrees of freedom (DOF) of the articulated vehicle. Three translational (longitudinal, lateral, and vertical) and three rotational (roll, pitch, and yaw) motions of the tractor sprung mass are considered. The semitrailer rotates freely with respect to the tractor in the yaw and pitch direction, but otherwise its motion is constrained by the fifth wheel. The roll and jounce (vertical) motions of the six wheel axles are also modeled and each of the twelve wheels spins independently. The longitudinal speed is controlled by a cruise controller, which is turned off once the brake input is applied. 
The original ArcSim model has 79 states - some of which only for animation purposes. 34 of these states, including the animation states and axle jounces and jounce rates, were found insignificant in the truck-rollover process and are therefore removed to form a simpler model [10], along which the linear analytical solution is obtained.

\subsection{The 1P worst-case algorithm}

Due to the fact that the truck rollover/jackknifing problem is formulated as a $1 \mathrm{P}$ problem, the analytical solution of the worst-case disturbance is obtained from [9]:

$$
\underline{w}(t)^{*}=-P^{-1} D^{T} K(t) \underline{x}(t)
$$

where the linearized truck dynamics are assumed to be $\underline{\dot{x}}(t)=A \underline{x}(t)+D \underline{w}(t)$, $\underline{x}(0)=0$ and the gain matrix $K(t)$ is solved from the Riccati-like equation

$$
\begin{aligned}
& \dot{K}(t)=-A^{T} K(t)-K(t) A+K(t) D P^{-1} D^{T} K(t)+Q \\
& K\left(t_{f}\right)=0
\end{aligned}
$$

In most applications, we found that the steady-state solution of Eq.(2), if it exists, is a good sub-optimal solution. The solution from Eq.(1) is then fed to the nonlinear TPBVP as the initial guess. The final worst-case disturbance is obtained from [11]:

$$
\underline{w}(t)=-P^{-1}\left[\frac{\partial f(t)}{\partial \underline{w}(t)}\right]^{T} \underline{\lambda}(t)
$$

where the costate $\underline{\lambda}$ is obtained from

$$
\underline{\dot{\lambda}}(t)^{T}=\underline{x}(t)^{T} Q-\underline{\lambda}(t)^{T} \frac{\partial f(t)}{\partial \underline{x}(t)} \underline{\lambda}\left(t_{f}\right)=0
$$

\subsection{Rollover simulation results}

First, we assume that only steering input is allowed (i.e., no braking inputs). When the vehicle speed is $60 \mathrm{~m} / \mathrm{h}$, it was found that the truck rolls over under a step steering of 160 degrees or higher (at the steering wheel), which translates to about 2.5 degrees at the front tires.

When the wheel steering angle is limited to \pm 130 degrees, it is no longer trivial to find a maneuver that will cause the truck to roll over. Figure 2 shows the vehicle responses under two input excitations: a 130-degree step and a 130-degree sinusoidal steering. The frequency of the sinusoidal input is selected to coincide with the natural 
frequency of the vehicle roll mode $(0.4 \mathrm{~Hz})$. The response of the vehicle under the sinusoidal excitation is interesting since the slalom test, frequently used to illustrate the rollover characteristics of passenger vehicles, is sinusoidal in nature. It is clear that the truck does not roll over under either maneuver and the maximum roll angle is only about 6 degrees. However, this does not imply that limiting the steering angle to 130 degrees would prevent rollover based on these simulation results. The safety of the vehicle can be guaranteed only if the worst steering input is identified.

By using the proposed algorithm, the worst-case steering input was identified and the vehicle response is shown in Figure 3. The worst-case steering switches between the limit values back and forth two times. The roll angle grows dramatically after the steering switches the last time and the vehicle finally rolls over.
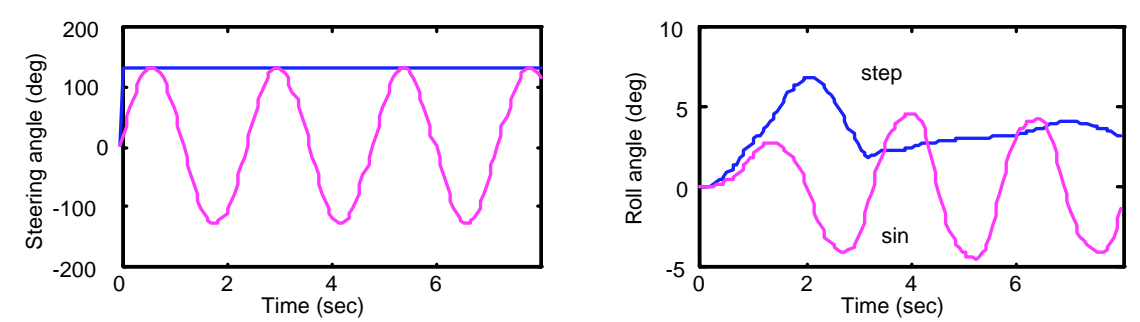

Fig. 2. Vehicle response to step and sinusoidal steering inputs (no braking).
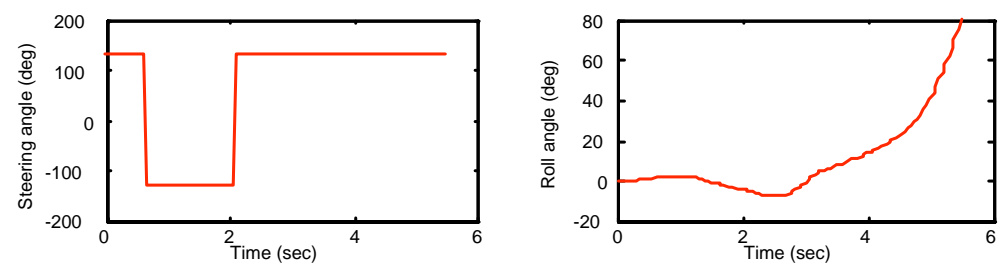

Fig. 3. Vehicle response to worst-case rollover maneuver (steering, no braking).

When there is more than one input to manipulate, no general guidelines are available to generate cooperating input signals. It is difficult to develop meaningful and representative maneuvers by intuition. Therefore, inputs are often generated in an ad hoc manner. One such coordination scheme has been proposed by Dugoff et al. [3] at UMTRI for the evaluation of passenger vehicles. The proposed maneuver applies steering that excites the vehicle consistently on one side (half sinusoidal, step, etc.). The steering is complemented by applying maximum braking pressure when the vehicle roll rate first becomes zero (i.e., the vehicle roll angle peaks on one side). The vehicle then starts to roll to the other side due to the suddenly reduced lateral acceleration. Once the roll rate becomes zero again then the brake is switched off. This "drastic maneuver" was applied in vehicle field tests and successfully rolled 
over a Chevy Corvair. In the simulations, the same idea was adopted and this "drastic maneuver" was applied to the truck.

The vehicle response to the corresponding "drastic maneuver" (with the steering limit of 29 degrees and the braking limit of 120 psi) is as shown in Figure 4. While the drastic maneuver is cleverly designed, it focuses on manipulating the brake. The true potential of the steering has not been fully explored. The maximum roll angle achieved is 1.1 degrees. Under the same steering and brake limits (29 degrees and $120 \mathrm{psi}$ ), the results of the worst-case maneuver are as shown in Figure 5. Similarly to the "drastic maneuver," in the worst-case maneuver, the brake switches on and off but the vehicle now steers only to one side. In about four seconds, the truck rolls over. It should be noted that the brake pressure switching does not quite correspond to the time when the vehicle roll rate is zero. Figure 6 is a picture from the ArcSim animator, which shows the truck rolling over in the worst-case maneuver. The simulation results clearly demonstrate the value of the worst-case methods, and how the results from such methods may differ from ad hoc methods.
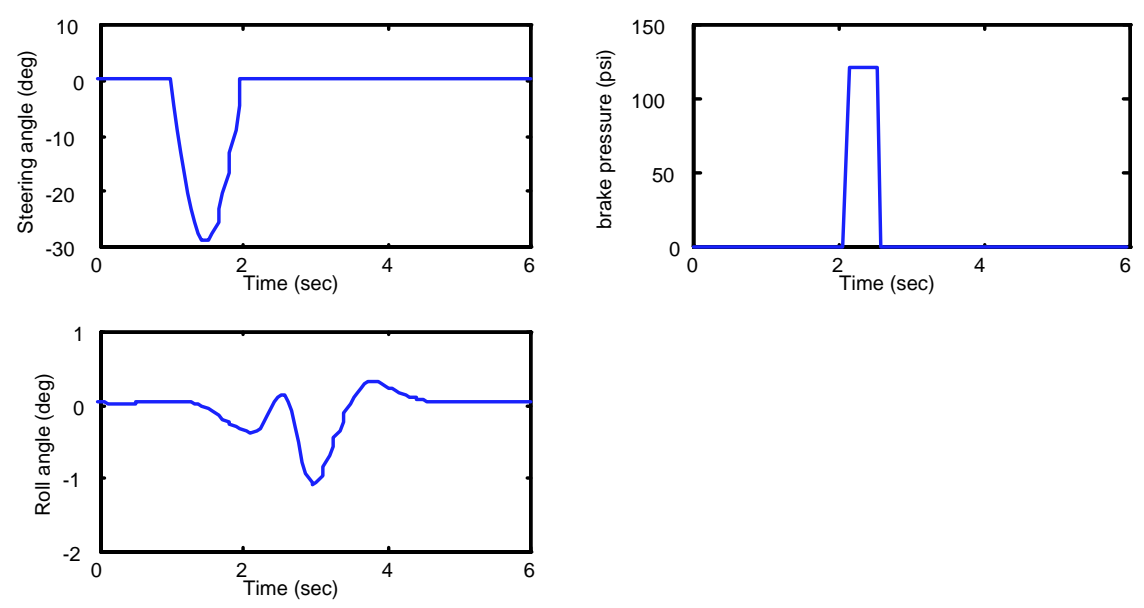

Fig. 4. Vehicle response under drastic maneuver.

As pointed out earlier, the purpose of the worst-case vehicle evaluation methodology is to better assess vehicle performance under extreme condition so that vehicle safety can be improved. Preliminary research results applying this methodology are presented below.

First, under the nominal vehicle load condition, and assuming that the brake pressure is limited to $120 \mathrm{psi}$, the maximum steering angle allowed without any possibility of rollover is listed in Table 1 . The maximum steering angle is presented under varying vehicle speed, tire/road friction coefficient and track width, and represent condition for guaranteed safe operation of this truck. If this table is constructed 

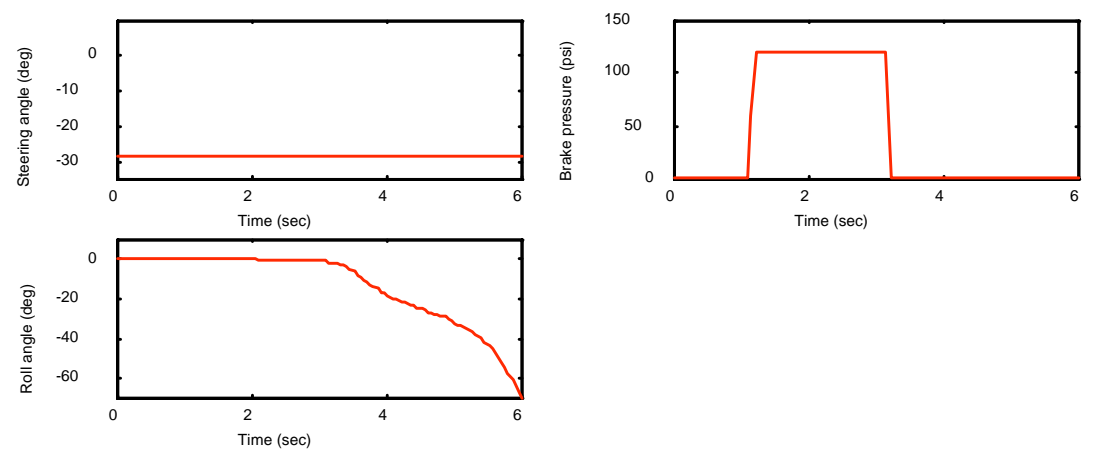

Fig. 5. Vehicle response to the worst-case maneuver for rollover (steering and braking).

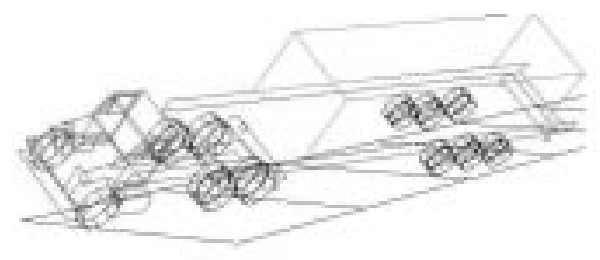

Fig. 6. Rollover of the articulated truck.

for several different trucks, their safety (in the worst-case sense) can be compared objectively.

\subsection{Jackknifing simulation results}

When the cost function is modified to maximize the articulation angle, the "worstcase" algorithm manipulates the steering and braking to create a large articulation angle (possibly result in jackknife). This objective was found to be not always achievable. When the road friction coefficient is high, the truck may roll over first before significant articulation angle is generated. When the road friction is low, it is possible to jackknife the truck even at a very low level of steering. For example, assuming that the road friction coefficient $\mu=0.4$, the truck can be jackknifed even when the steering angle is as low as 4 degrees (see Fig. 8). To generate jackknifing, it is necessary to apply heavy braking for an extended period of time.

Steering plays only a secondary role. A large articulation angle is mainly generated from the inverted pendulum nature of the articulated vehicle under adverse brake force distribution. The truck jackknifing can also be visualized from the ArcSim animator (Fig. 9). 
Table 1. Maximum Steering Angle without Rollover

\begin{tabular}{cccc}
\hline & & track width & \\
& $-10 \%$ & baseline & $+10 \%$ \\
\hline vehicle speed $60 \mathrm{mph}$ & & & 29 \\
\hline$\mu=0.8$ & 24 & 28 & 16 \\
$\mu=0.6$ & 14 & 15 & 12 \\
$\mu=0.4$ & 10 & 12 & 84 \\
\hline vehicle speed $50 \mathrm{mph}$ & & & 44 \\
$\mu=0.8$ & 70 & 77 & 25 \\
$\mu=0.6$ & 33 & 37 & 170 \\
$\mu=0.4$ & 20 & 22 & 104 \\
\hline vehicle speed $40 \mathrm{mph}$ & & & 90 \\
\hline$\mu=0.8$ & 124 & 154 & \\
\hline$\mu=0.6$ & 84 & 94 & \\
\hline
\end{tabular}
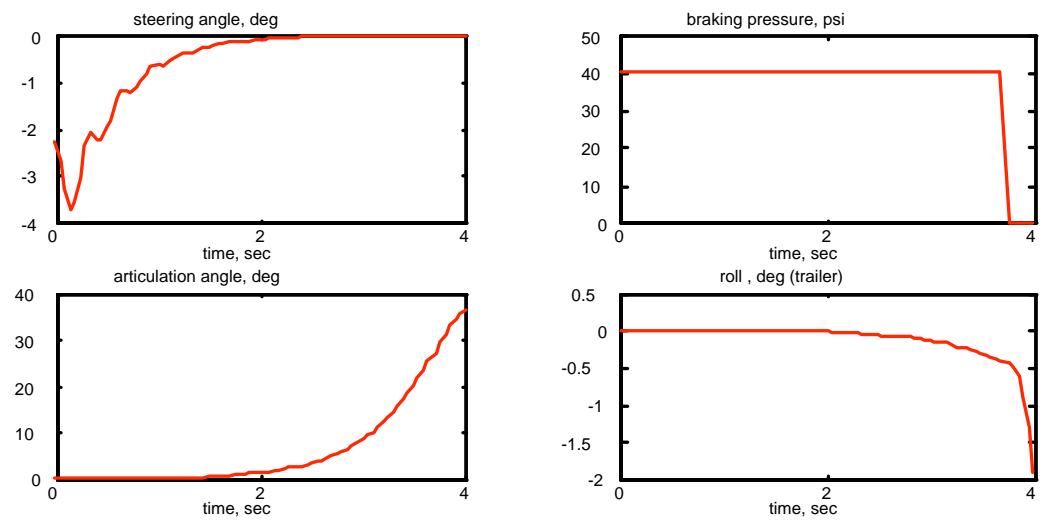

Fig. 7. Vehicle Responses under the Worst-Case Jackknifing Maneuver.

Fig. 8. Jackknifing of the Truck. 


\section{ACTIVE YAW CONTROL EVALUATIONS}

In this section, the worst-case evaluation of an AYC system is used as an example two-player worst-case evaluation problem. The worst-case AYC evaluation problem is officially stated below:

Given a nonlinear vehicle dynamic model $\underline{\dot{x}}=f(\underline{x}, \underline{u}, \underline{w}, t)$, where the disturbance input $\underline{w}$ includes front wheel steering angle $\delta$ and brake pedal command $p$. The control input $\underline{u}$ includes the ABS pressure command $p_{A B S}$ and AYC pressure command $p_{A Y C}$. The final brake pressure to the vehicle wheels depend on the three pressure command signals. Assuming that the control algorithms of the $A B S$ and AYC modules are known. Find, within saturation bound $-\delta_{\max } \leq \delta \leq \delta_{\max }$ and $0 \leq p \leq p_{\max }$, the time signal $\underline{w}(t)$ which maximizes a cost function $J(\underline{x}, \underline{w}, t)=\int_{0}^{T} \underline{x}^{T}(t) Q \underline{x}(t)+\underline{u}^{T}(t) R \underline{u}(t)-\underline{w}^{T}(t) P \underline{w}(t) d t$. The matrix $Q$ is selected such that the vehicle side slip angle is maximized.

\subsection{Vehicle simulation model}

Nomenclature

$a_{x}\left(a_{y}\right)$ : vehicle longitudinal (lateral) acceleration
$\beta$ : vehicle side slip angle
$\delta$ : steering angle of the front tires

$\mathrm{F}_{\mathrm{xi}}\left(\mathrm{F}_{\mathrm{yi}}\right)\left(\mathrm{F}_{\mathrm{zi}}\right)$ : longitudinal (lateral)(vertical) force of tire i, i=1..4

$\mathrm{I}_{\mathrm{Z}}$ : yaw moment of inertia of the vehicle.

$\mathrm{I}_{\omega \mathrm{i}}$ : moment of inertia of the $\mathrm{i}^{\text {th }}$ wheel, $\mathrm{i}=1 . .4$

$l_{1}\left(l_{2}\right)$ : distance C.G. to front (rear) axle L: wheel base $\left(=l_{1}+l_{2}\right)$

$\mathrm{M}$ : vehicle total mass

$\mathrm{M}_{\mathrm{S}}$ : vehicle sprung mass

r: yaw rate

$\mathrm{S}_{\mathrm{b} 1}\left(\mathrm{~S}_{\mathrm{b} 2}\right)$ : track width of front (rear) axle

u: longitudinal velocity

v: lateral velocity

V: resultant vehicle velocity

The architecture of the vehicle simulation model used in this paper is illustrated in Figure 10. Two assumptions were made: (i) AYC only increases the brake pressure on one of the front wheels. It never reduces brake pressure; (ii) The overall brake pressure applied to the tires is the sum of the three brake command signals from the ABS $\left(P_{A B S}\right)$, AYC $\left(p_{A Y C}\right)$, and the driver $\left(P_{d r v}\right)$. The ABS releases brake pressure to prevent wheel lock-up due to excessive braking from the driver and the AYC. 


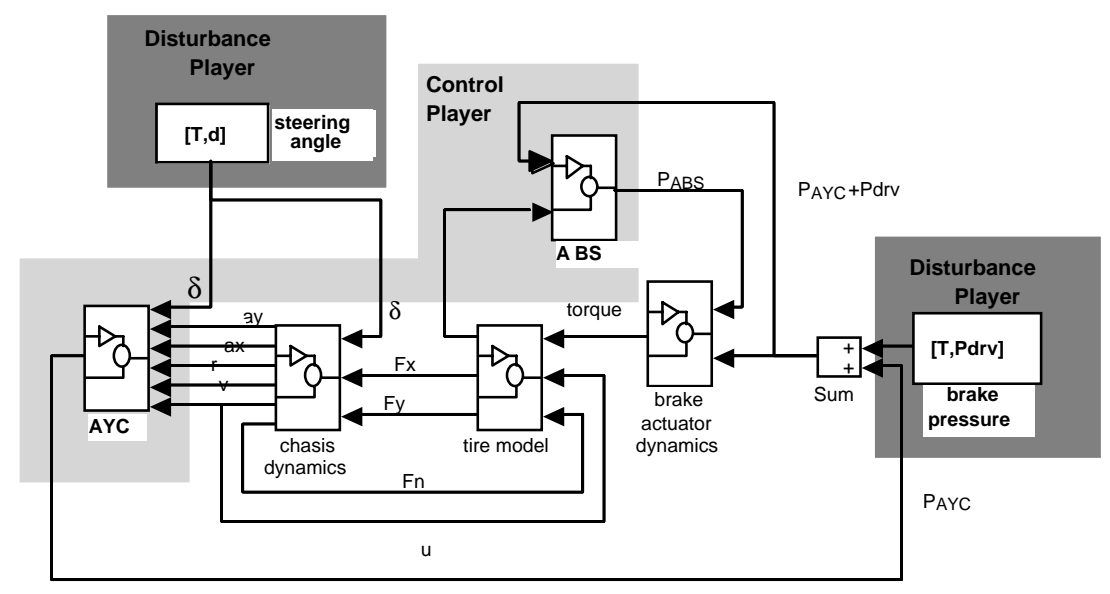

Fig. 9 Architecture of the Vehicle Model.

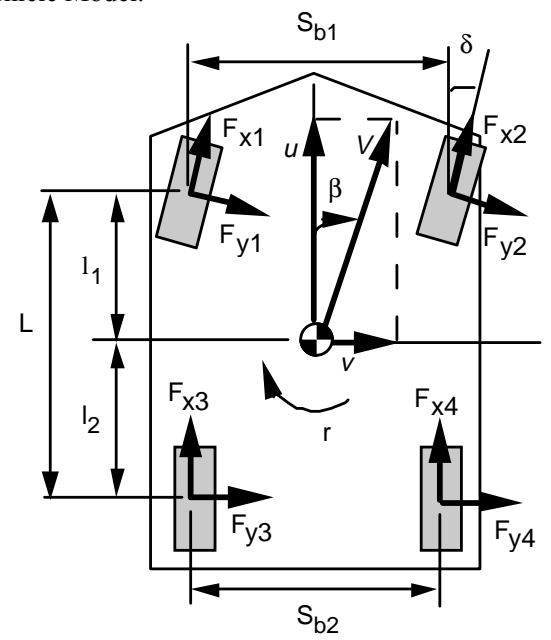

Fig. 10. The Yaw Plane Vehicle Model.

The AYC control algorithm is usually designed based on vehicle yaw plane models $([4,12])$, i.e., the pitch, roll, and vertical motions are neglected. In this paper, a yaw plane vehicle model of 14 state variables, shown in Figure 11, is created to describe the dynamics of a passenger car. The vehicle sprung mass has three DOF (longitudinal, lateral, and yaw) and thus needs six state variables. Four states are defined for the wheel rotations. The last four states are the brake torque applied at the four wheels.

The equations governing the three DOF vehicle rigid body motion are

$M(\dot{u}-v r)=F_{x 1} \cos \delta-F_{y 1} \sin \delta+F_{x 2} \cos \delta-F_{y 2} \sin \delta+F_{x 3}+F_{x 4}$ 


$$
\begin{gathered}
M(\dot{v}+u r)=F_{x 1} \sin \delta+F_{y 1} \cos \delta+F_{x 2} \sin \delta+F_{y 2} \cos \delta+F_{y 3}+F_{y 4} \\
I_{z} \dot{r}=l_{1}\left(F_{y 1}+F_{y 2}\right) \cos \delta-l_{2}\left(F_{y 3}+F_{y 4}\right)-\frac{S_{b 1}}{2}\left(F_{y 1}-F_{y 2}\right) \sin \delta \\
+\frac{S_{b 1}}{2}\left(F_{x 1}-F_{x 2}\right) \cos \delta+\frac{S_{b 2}}{2}\left(F_{x 3}-F_{x 4}\right)+l_{1}\left(F_{x 1}+F_{x 2}\right) \sin \delta
\end{gathered}
$$

Although the vehicle roll motion is neglected, the load transfer due to (steadystate) roll is approximated and included in the dynamic equations. Based on this steady-state approximation, tire normal forces are calculated. The tire radius is then obtained from

$$
R_{i}=R_{o i}-\frac{F_{z i}}{K_{z i}} \quad \text { for } \mathrm{i}=1, . ., 4
$$

where $K_{z i}$ is the tire vertical stiffness and $\mathrm{R}_{\mathrm{Oi}}$ is the original radius of the $\mathrm{i}^{\text {th }}$ tire. The wheel angular velocities, $\omega_{i}$, are governed by the following dynamic equation:

$$
\dot{\omega}_{i}=\frac{-T_{b i}-F_{x i} R_{i}}{I_{\omega i}} \quad \text { for } \mathrm{i}=1, . ., 4
$$

where $T_{b i}$ is the brake torque and $I_{\omega i}$ is the effective wheel rotational inertia (including power-train inertia).

The nonlinear behavior of tires is very important in the AYC simulations since the tires may operate in saturated regions. In this paper, a Magic Formula tire model [13] is used. The tire longitudinal and lateral forces are calculated from

$$
\begin{aligned}
& F_{x o}=D_{x} \sin \left(C_{x} \tan ^{-1}\left(B_{x} \Phi_{x}\right)\right)+S_{v x} \\
& F_{y o}=D_{y} \sin \left(C_{y} \tan ^{-1}\left(B_{y} \Phi_{y}\right)\right)+S_{v y}
\end{aligned}
$$

where

$$
\begin{aligned}
& \Phi_{x}=\left(1-E_{x}\right)\left(\lambda+S_{h x}\right)+\frac{E_{x}}{B_{x}} \tan ^{-1}\left(B_{x}\left(\lambda+S_{h x}\right)\right) \\
& \Phi_{y}=\left(1-E_{y}\right)\left(\alpha+S_{h y}\right)+\frac{E_{y}}{B_{y}} \tan ^{-1}\left(B_{y}\left(\alpha+S_{h y}\right)\right)
\end{aligned}
$$

The coefficients, $B_{x}, B_{y}, C_{x}, C_{y}, D_{x}, D_{y}, E_{x}, E_{y}, S_{h x}, S_{h y}, S_{V x}$, and $S_{V y}$ dependent on tire normal forces, and were found experimentally to be [14]:

$$
B_{y}=0.22+\frac{5200-F_{z}}{40000} \quad C_{y}=1.26-\frac{F_{z}-5200}{32750} \quad D_{y}=-0.00003 F_{z}^{2}+1.0096 F_{z}-22.73
$$


$E_{y}=-1.6$

$S_{h y}=0$

$S_{v y}=0$

For $\lambda>0$

$$
\begin{array}{lll}
B_{x}=22+\frac{F_{z}-1940}{645} & C_{x}=1.35-\frac{F_{z}-1940}{16125} & D_{x}=2000+\frac{F_{z}-1940}{0.956} \\
E_{x}=-3.6 & S_{h x}=0 & S_{v x}=0
\end{array}
$$

For $\lambda \leq 0$

$$
\begin{array}{ll}
B_{x}=22+\frac{F_{z}-1940}{430} & C_{x}=1.35-\frac{F_{z}-1940}{16125} D_{x}=1750+\frac{F_{z}-1940}{0.956} \\
E_{x}=0.1 & S_{h x}=0
\end{array}
$$

Due to the fact that both steering and braking inputs can be quite large, the Magic Formula needs to be modified to include combined-slip conditions. This is achieved by the following procedure: (i) Compute the longitudinal and lateral forces based on Eqs. (14) and (15); (ii) Define normalized slip $\lambda^{*}, \alpha^{*}$ and the combined slip $\sigma^{*}$ as $\lambda^{*} \equiv \frac{\lambda}{\lambda_{m}}, \alpha^{*} \equiv \frac{\alpha}{\alpha_{m}}$ and $\sigma^{*} \equiv\left[\left(\lambda^{*}\right)^{2}+\left(\alpha^{*}\right)^{2}\right]^{0.5}$ where $\lambda_{\mathrm{m}}, \alpha_{\mathrm{m}}$ are the values when $\mathrm{F}_{\mathrm{X}}$ and $\mathrm{F}_{\mathrm{y}}$ reach the peak values, respectively; and (iii) The modified forces are then obtained from $F_{x}=\frac{\lambda^{*}}{\sigma^{*}} F_{x o}\left(\sigma^{*}, F_{z}\right)$ and $F_{y}=\frac{\alpha^{*}}{\sigma^{*}} F_{y o}\left(\sigma^{*}, F_{z}\right)$, respectively.

The brake model used in this research consists of a first-order lag and a pure time delay:

$$
\dot{T}_{b}=\frac{C_{1}}{\tau_{2}} P_{b}\left(t-\tau_{1}\right)
$$

where $\mathrm{T}_{b}$ is the braking torque, $\mathrm{C}_{1}$ is the steady-state gain from brake pressure to brake torque, and $\mathrm{P}_{\mathrm{b}}$ is the brake pressure applied to the actuator, and is the sum of the inputs from the driver $\left(P_{d r v}\right)$, the AYC $\left(P_{A Y C}\right)$, and the ABS $\left(P_{A B S}\right)$. A simple diagram of the brake model is shown in Figure 12.

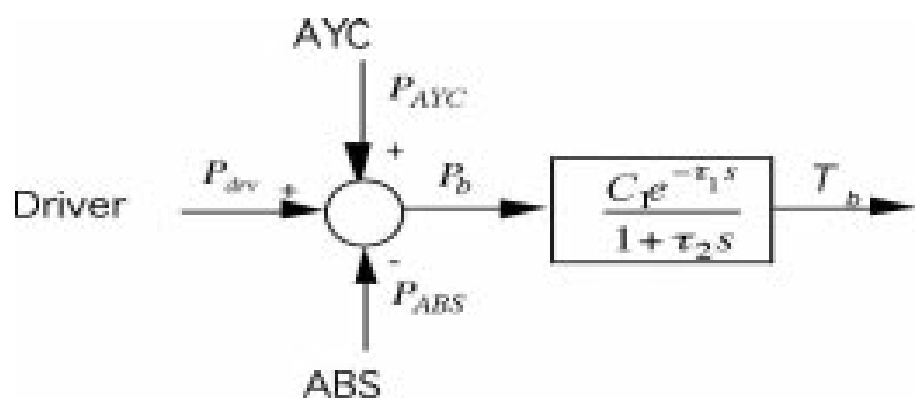

Fig. 11. The Brake Model. 
A simple rule-based ABS is used in this research. The tire slip ratio, $\lambda$, is assumed to be known to simplify the design. The following ABS algorithm is designed to keep $\lambda$ in a range of $\left[\lambda_{\min }, \lambda_{\max }\right]$

$$
\begin{aligned}
& \text { if }|\lambda|<\lambda_{\text {min }} \text { then } \Delta P_{A B S}=-C_{A B S 1}\left(\lambda_{\text {min }}-|\lambda|\right)\left|P_{d r v}+P_{A Y C}\right| \\
& \text { else if }|\lambda|>\lambda_{\text {max }} \text { then } \Delta P_{A B S}=C_{A B S 2}\left(|\lambda|-\lambda_{\text {max }}\right)\left|P_{d r v}+P_{A Y C}\right| \\
& \text { else if }|\lambda|>\lambda_{m} \text { then } \Delta P_{A B S}=C_{A B S 3} \Delta P_{A B S} \\
& P_{A B S}(k)=P_{A B S}(k-1)+\Delta P_{A B S}
\end{aligned}
$$

where $C_{A B S 1}$ and $C_{A B S 2}$ are properly selected control gains. When $\lambda$ is outside of the proper range, the ABS will modify the brake pressure in proportion to the brake pressure and the deviation of the desired tire slip. When the tire slip is in the desired range $\left[\lambda_{\min }, \lambda_{\max }\right]$ and greater than $\lambda_{m}$, which corresponds to the peak tire longitudinal force, the ABS pressure is gradually scaled back by the factor, $C_{A B S 3}$ $\left(0<C_{A B S 3}<1\right)$. It should be noted that the ABS only releases the brake pressure. Therefore, $P_{A B S}$ is always positive and is between zero and $\left|P_{d r v}+P_{A Y C}\right|$.

The AYC design used in this paper is a yaw-rate feedback approach [6]. Figure 13 shows the block diagram of the AYC sub-system. When the vehicle side slip angle, $\beta$, is greater than a threshold, AYC is activated. The magnitude of AYC braking is calculate from

$$
\Delta P_{A Y C}=C_{A Y C 1}\left|r-r_{d}\right|+C_{A Y C 2}\left|\beta+C_{A Y C 3} \dot{\beta}\right|
$$

where $r_{d}$ is the desired yaw rate, which is estimated from steering angle and road friction coefficient. The differential braking from AYC is applied only to the front tires. When $\beta$ is positive and greater than a threshold, the AYC will activate the front right tire. Similarly, if $\beta$ is negative and less than a threshold, the brake pressure at the front left will be increased.

\subsection{The $2 \mathrm{PP}$ worst-case algorithm}

The AYC evaluation example is formulated as a $2 \mathrm{PP}$ problem, the analytical solution of the worst-case disturbance is obtained from [9]:

$$
\underline{w}^{*}(t)=P^{-1} D^{T}\left[K(t) \underline{x}(t)+\int_{0}^{t_{l a}} F_{1}(t, l) \underline{u}(t+l) d l\right]
$$




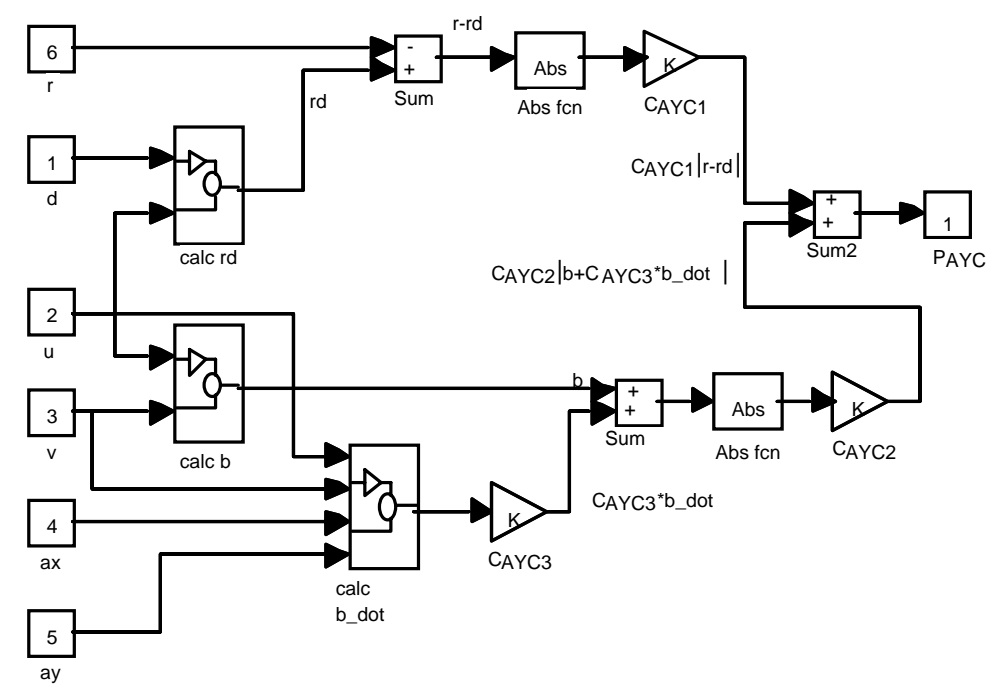

Fig. 12. The AYC Sub-system.

The values of the vehicle and AYC parameters are shown in Table 2 for reference.

Table 2. Parameters of the Vehicle Model.

\begin{tabular}{llllll}
\hline parameter & value & unit & parameter & value & unit \\
$\mathrm{M}$ & 1573 & $\mathrm{Kg}$ & $\mathrm{K}_{\mathrm{zi}}, \mathrm{i}=1, . ., 4$ & 190632 & $\mathrm{~N} / \mathrm{m}$ \\
$\mathrm{M}_{\mathrm{S}}$ & 1415.7 & $\mathrm{Kg}$ & $\mathrm{K}_{\mathrm{ax} 1}$ & 71485 & $\mathrm{~N}$ \\
$\mathrm{I}_{\mathrm{Z}}$ & 2783 & $\mathrm{Kg}-\mathrm{m}^{2}$ & $\mathrm{~K}_{\mathrm{ax} 2}$ & 71485 & $\mathrm{~N}$ \\
$\mathrm{I}_{\omega \mathrm{i}}, \mathrm{i}=1,2$ & 5 & $\mathrm{Kg}^{2} \mathrm{~m}^{2}$ & $\mathrm{C}_{1}$ & 4 & $\mathrm{~N}-\mathrm{m} / \mathrm{psi}$ \\
$\mathrm{I}_{\omega \mathrm{i}}, \mathrm{i}=3,4$ & 2 & $\mathrm{Kg}-\mathrm{m}^{2}$ & $\tau_{1}$ & 0.05 & $\mathrm{sec}$ \\
$\mathrm{H}_{\mathrm{CG}}$ & 0.478 & $\mathrm{~m}$ & $\tau_{2}$ & 0.05 & $\mathrm{sec}$ \\
$\mathrm{H}_{\mathrm{ax} 1}$ & 0.187 & $\mathrm{~m}$ & $\mathrm{C}_{\mathrm{AYC} 1}$ & 120 & \\
$\mathrm{H}_{\mathrm{ax} 2}$ & 0.187 & $\mathrm{~m}$ & $\mathrm{C}_{\mathrm{AYC} 2}$ & 800 & \\
$\mathrm{H}_{\mathrm{r}}$ & 0.3 & $\mathrm{~m}$ & $\mathrm{C}_{\mathrm{AYC} 3}$ & 1 & \\
$\mathrm{~S}_{\mathrm{b} 1}$ & 1.45 & $\mathrm{~m}$ & $\mathrm{C}_{\mathrm{ABS} 1}$ & 0.1 & \\
$\mathrm{~S}_{\mathrm{b} 2}$ & 1.45 & $\mathrm{~m}$ & $\mathrm{C}_{\mathrm{ABS} 2}$ & 0.1 & \\
$\boldsymbol{l}_{1}$ & 1.034 & $\mathrm{~m}$ & $\mathrm{C}_{\mathrm{ABS} 3}$ & 0.98 & \\
$\boldsymbol{l}_{2}$ & 1.491 & $\mathrm{~m}$ & $\lambda_{\min }$ & 0.05 & \\
$\mathrm{R}_{\mathrm{oi}}, \mathrm{i}=1, . ., 4$ & 0.3044 & $\mathrm{~m}$ & $\lambda_{\max }$ & 0.15 & \\
\hline
\end{tabular}


where the linearized vehicle dynamics are assumed to be $\underline{\dot{x}}(t)=A \underline{x}(t)+B \underline{u}(t)+D \underline{w}(t), \underline{x}(0)=0$ and the feedback and feedforward gain matrices $\bar{K}(t)$ and $F_{1}(\bar{t}, l)$ are solved from

$$
\begin{aligned}
& \dot{K}(t)=-A^{T} K(t)-K(t) A+K(t) D P^{-1} D^{T} K(t)+Q \quad K(t f)=0 \\
& \frac{\partial F_{1}(t, l)}{\partial t}=\frac{\partial F_{1}(t, l)}{\partial l}-\left(K(t) D P^{-1} D^{T}+A^{T}\right) F_{1}(t, l)-F_{1}\left(t, t_{l a}\right) R^{-1} K_{u}\left(t, l, t_{l a}\right) \\
& F_{1}(t, 0)=K(t) B \\
& \frac{\partial K_{u}\left(t, l_{1}, l_{2}\right)}{\partial t}=\frac{\partial K_{u}\left(t, l_{1}, l_{2}\right)}{\partial l_{1}}+\frac{\partial K_{u}\left(t, l_{1}, l_{2}\right)}{\partial l_{2}}-F_{1}(t, l)^{T} D P^{-1} D^{T} F_{1}(t, l) \\
& K_{u}(t, 0, l)=B^{T} F_{1}(t, l)
\end{aligned}
$$

The solution of Eq.(18) is then fed to the nonlinear TPBVP as the initial guess. A discrete-time TPBVP is formulated because it is easier to implement. Since the control law is completely known and delayed in time due to actuator lag, the vehicle dynamic equation is rewritten as $\underline{x}_{e}(k+1)=f\left(\underline{x}_{e}(k), \underline{x}_{e}(k-r) ; \underline{w}(k)\right)$, where the delayed control signal $u(t)$ is defined as part of the augmented state $\underline{x}_{e}$. The final worst-case disturbance is then obtained from [15]:

$$
\left[\frac{\partial f(\underline{x}(k), \underline{x}(k-r) ; \underline{w}(k))}{\partial \underline{w}(k)}\right]^{T} \underline{\lambda}(k)+\eta[P \underline{w}(k)]=0
$$

where the costate $\underline{\lambda}$ is obtained from

$$
\begin{aligned}
& \underline{\lambda}(k-1)=\left[\frac{\partial f(\underline{x}(k), \underline{x}(k-r) ; \underline{w}(k))}{\partial \underline{x}(k)}\right]^{T} \underline{\lambda}(k) \\
& +\left[\frac{\partial f(\underline{x}(k+r), \underline{x}(k) ; \underline{w}(k+r))}{\partial \underline{x}(k)}\right]^{T} \underline{\lambda}(k+r)-\eta[Q \underline{x}(k)] k=0, \ldots, N-1 \\
& \underline{\lambda}(k)=0, k \in[N-1, N+r-1]
\end{aligned}
$$

The variable $\eta$ is usually set to 1 if the constraint qualification holds ([16]).

\subsection{AYC simulation results}

The effectiveness of the AYC is first examined under three standard maneuvers: step steering, brake in a J-turn, and double lane changes. The vehicle speed and steering/braking inputs were selected such that in some cases the vehicle without AYC 
will spin out. The AYC design presented in the previous section was found to stabilize the vehicle under all these simulations. Representative simulation results under a double lane change maneuver are shown in Figure 14. It can be seen that the vehicle without AYC lost control when the steering direction is reversed. The vehicle with AYC, however, remains stable.

without AYC
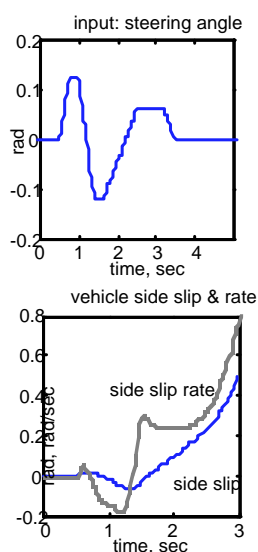

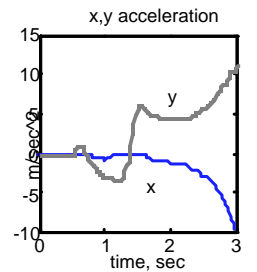

yaw rate

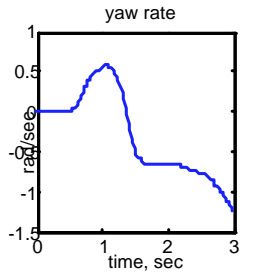

with AYC
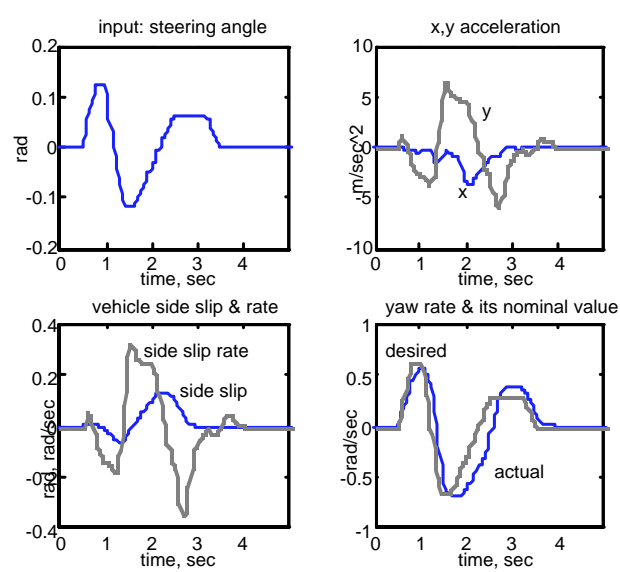

Fig. 13. Vehicle response under a double lane-change maneuver.

Figure 15 shows the vehicle, equipped with AYC, under two different maneuvers. Under the brake in a J-turn maneuver, the steering and braking input limits are $0.05 \mathrm{rad}$ and $300 \mathrm{psi}$, respectively. The vehicle remains stable under this severe maneuver. Under the same input limits, the worst-case steering/braking maneuver identified by the proposed algorithm generates a vehicle side slip angle as large as 60 degrees.

\section{CONCLUSIONS}

A worst-case vehicle evaluation methodology was developed to generate extreme input excitations for vehicle systems. Worst-case inputs are important because they are necessary to assess performance of vehicles or active-safety vehicle control systems. In this paper, two design examples were presented. For the truck example, it was found that a fully loaded articulated truck can roll over at a much reduced steering level if the driver applies a poorly-timed pulse braking. A systematic procedure based on the worst-case method was suggested which can be used to measure vehicle rollover propensity more objectively. 
Brake in a J-turn

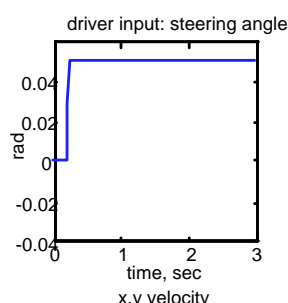

$x, y$ velocity
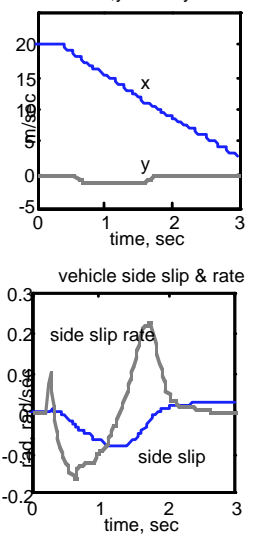

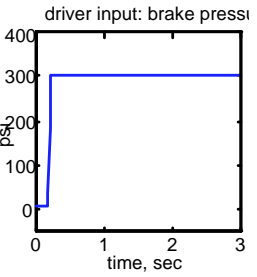

$x, y$ acceleration
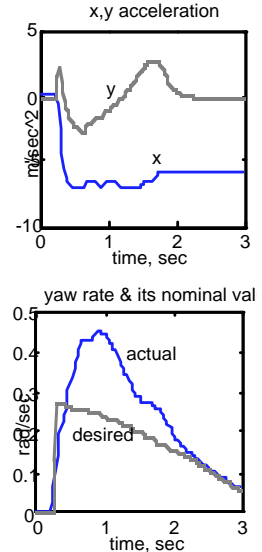

Worst-case maneuver

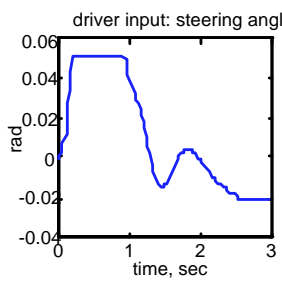

$\mathrm{x}, \mathrm{y}$ velocity
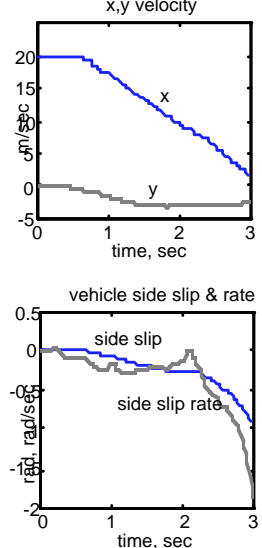

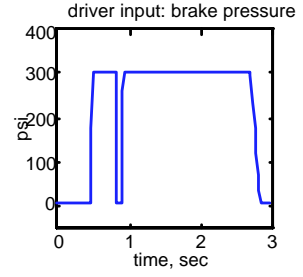

$x, y$ acceleration
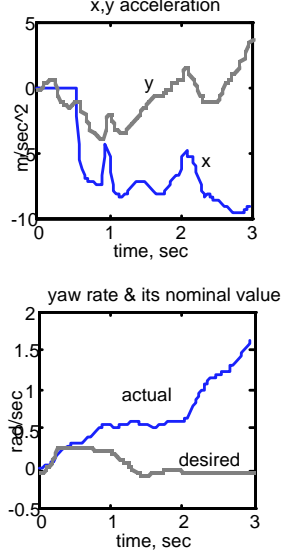

Fig. 14. Response of an AYC vehicle under two maneuvers.

For the AYC evaluation example, it was found that an AYC could behave extremely well under standard evaluation maneuvers (step steering, double lane change, brake in a J-turn) but large side slip angle and yaw rate can be generated under a worst-case maneuver at similar input levels. Currently, we are exploring the possibility of integrating the worst-case methodology with an iterative AYC design process.

\section{ACKNOWLEDGMENT}

This research was supported by the U.S. Army TARDEC under the contract DAAE07-94-C-R094.

\section{REFERENCES}

1. Winkler, C.B., Fancher, P.S., MacAdam, C.C., Parametric Analysis of Heavy Duty Truck Dynamic Stability, Volume 1 - Technical Report, Transportation Research Institute, University of Michigan, DOT HS-806-411, March 1983. 
2. Winkler, C.B., Karamihas, S.M., Bogard, S.E., "Roll-stability performance of heavy-vehicle suspensions," Proceedings of the 1992 International Truck and Bus Meeting and Exposition. Toledo, OH. SAE paper\#922426.

3. Dugoff, H., Ervin, R. D., Segel, L., "Vehicle Handling Test Procedures", Transportation Research Institute, University of Michigan, Report Contract No. FH-11-7297, November 1970.

4. Yamamoto, M., Inagaki, S., and Kushiro, I., "Improvement of Vehicle stability in Critical Cornering by Active Brake Control," Toyota Technical Review, 45(1) 64-69, 1995

5. Koibuchi, K., Yamamoto, M., Fukada, Y., and Inagaki, S., "Vehicle Stability Control in Limit Cornering by Active Brake," SAE Paper No. 960487, 1996.

6. Van Zanten, A. T., Erhardt, R., and Pfaff, G., "VDC, the Vehicle Dynamics Control System of BOSCH," SAE Paper No. 950759, 1995.

7. Alberti, V. and Babbel, E., "Improved Driving Stability by Active Braking of the Individual Wheels," Proceedings of the International Symposium on Advanced Vehicle Control, pp. 717-732, 1996.

8. Ma, W. and Peng, H., "Worst-case Evaluation Methods for Vehicle Control Systems," Proceedings of the 1996 ASME International Congress and Exposition, Atlanta, GA, November 1996.

9. Ma, W. and Peng, H., "A Worst-case Evaluation Method for Dynamic Systems," to appear in the ASME Journal of Dynamic Systems, Measurement and Control.

10. Ma, W. Worst-Case Evaluation Methods For Vehicles And Vehicle Control Systems, Ph.D. dissertation, University of Michigan, May 1998.

11. Bryson, A. E., and Ho, Y. C., Applied Optimal Control, Hemisphere Publishing, 1975.

12. Yasui, Y., Tozu, K., Hattori, N., and Sugisawa, M. "Improvement of Vehicle Directional Stability for Transient Steering Maneuvers Using Active Brake Control," SAE Paper No. 960485, 1996.

13. Bakker, E., Nyborg, L., Pacejka, H. B., " Tyre Modelling for Use in Vehicle Dynamic Studies," SAE Paper No. 870421, 1987.

14. Peng, H., Vehicle Lateral Control for Highway Automation, Ph.D. Dissertation, University of California, Berkeley, 1992.

15. Mariani, L. and Nicoletti, B., "Optimal Discrete System with Pure Delays," IEEE Transactions on Automatic Control, Vol. 18, No. 3, pp. 311-313, 1973.

16. Pearson, J. B. Jr. and Sridhar, R., "A Discrete Optimal Control Problem," IEEE Transactions on Automatic Control, Vol. AC-11, pp. 171-174, 1966. 